\title{
Agua potable para uso doméstico: análisis del ciclo de vida y de escenarios hipotéticos de manejo ambiental para la ciudad de Pereira, Colombia*
}

\author{
Manuel Varón-Hoyos* \\ Álvaro Restrepo-Victoria ${ }^{* * *}$ \\ Jhoniers Guerrero-Erazo ${ }^{* * * *}$
}

Recibido: 13/10/2017 • Aceptado: 20/11/2018

https://doi.org/10.22395/rium.v18n35a2

\begin{abstract}
Resumen
En este estudio se caracterizan los impactos ambientales asociados al ciclo de vida de $1 \mathrm{~m}^{3}$ de agua potable en Pereira, Colombia. Se analizaron cuatro escenarios: un escenario base y tres escenarios ambientales hipotéticos. Se describió el ciclo de vida, se hizo el inventario de flujos de materia y energía y se establecieron los impactos ambientales de punto medio y punto final del escenario base mediante la aplicación del método Impact 2002+, asimismo, se definieron los impactos ambientales de tres escenarios hipotéticos.
\end{abstract}

Los resultados determinaron que los procesos relacionados con la producción de agua potable generaron la mayor cantidad de impactos negativos del ciclo (75-100 \%) en 12 de las 15 categorías de punto medio en el escenario base. Asimismo, en dicho escenario el agua potable contribuyó al $56 \%$ del impacto de punto final. Por otra parte, impulsar la reducción del $20 \%$ en el consumo de agua junto con el tratamiento de las aguas residuales sería el mejor escenario futuro, tanto para reducir impactos de punto medio, como para reducir los de punto final.

Palabras clave: tratamiento del agua; agua potable; aguas residuales; ciclo de vida.

* Artículo derivado de la investigación Agua potable para uso doméstico en la ciudad de Pereira: análisis de ciclo de vida y escenarios de manejo ambiental, realizada como trabajo de grado de la Maestría en Ecotecnología de la Universidad Tecnológica de Pereira, entre octubre del 2013 y julio del 2015. Investigación financiada con recursos propios.

** Magíster en Ecotecnología, consultor y miembro del Grupo de Investigación en Gestión Ambiental Territorial (GAT) de la Facultad de Ciencias Ambientales, Universidad Tecnológica de Pereira, Colombia. Correo electrónico: manuelvaron@utp.edu.co. Orcid: https://orcid.org/0000-0003-0761-143X

*** Doctor en Ingeniería y profesor titular de la Facultad de Ingeniería Mecánica. Miembro del Grupo de Investigación en Gestión Energética, Universidad Tecnológica de Pereira, Colombia. Correo electrónico: arestrep@utp.edu. co. Orcid: https://orcid.org/0000-0002-0565-3532

***** Doctor en Ingeniería y profesor titular de la Facultad de Ciencias Ambientales. Miembro del Grupo de Investigación en Agua y Saneamiento, Universidad Tecnológica de Pereira, Colombia. Correo electrónico: jhguerre@ utp.edu.co. Orcid: https://orcid.org/0000-0002-0243-2320 


\title{
Drinking Water for Domestic Use: Life Cycle Analysis and Hypothetical Environmental Management Scenarios for the City of Pereira, Colombia
}

\begin{abstract}
In this study, the environmental impacts associated with the life cycle of $1 \mathrm{~m}^{3}$ of drinking water in Pereira, Colombia were characterized. Four scenarios were analyzed: a baseline scenario and three hypothetical environmental scenarios. The life cycle was described, the inventory of matter and energy flows was made, and the environmental impacts of the midpoint and endpoint of the baseline scenario were established using the Impact 2002+ method, the environmental impacts of three hypothetical scenarios were defined also.

The results determined that the processes related to the production of drinking water generated most of the negative impacts of the cycle (75-100\%) in 12 of the 15 categories of midpoint in the baseline scenario. Likewise, in this scenario, drinking water contributed $56 \%$ of the endpoint impact. On the other hand, promoting a $20 \%$ reduction in water consumption along with the treatment of wastewater would be the best future scenario, both to reduce mid-point impacts and to reduce end-point impacts.
\end{abstract}

Keywords: water treatment; drinking water; wastewater; life cycle.

\section{Água potável para uso doméstico: análise do ciclo de vida e de cenários hipotéticos de gestão ambiental para a cidade de Pereira, Colômbia}

\section{Resumo}

Neste estudo, caracterizamos os impactos ambientais associados ao ciclo de vida de $1 \mathrm{~m}^{3}$ de água potável em Pereira, na Colômbia. Quatro cenários foram analisados: um cenário-base e três cenários ambientais hipotéticos. Descreveu-se o ciclo de vida, fez-se um inventário de fluxos de matéria e energia e estabeleceram-se os impactos ambientais orientados a ponto médio e a ponto final do cenário-base por meio da aplicação do método Impact 2002+. De igual forma, definiram-se os impactos ambientais de três cenários hipotéticos.

Os resultados determinaram que os processos relacionados com a produção de água potável geraram a maior quantidade de impactos negativos do ciclo $(75-100 \%) \mathrm{em}$ 12 das 15 categorias de ponto médio no cenário-base. Do mesmo modo, em tal cenário a água potável contribuiu em $56 \%$ do impacto de ponto final. Por outro lado, incentivar a redução de $20 \%$ do consumo de água juntamente com o tratamento das águas residuais seria o melhor cenário futuro - tanto para reduzir impactos de ponto médio quanto para reduzir os de ponto final.

Palavras-chave: tratamento da água, água potável, águas residuais, ciclo de vida. 


\section{INTRODUCCIÓN}

A medida que pasa el tiempo se conocen mejor las repercusiones del consumo urbano en el ciclo del agua, entre las que destacan la escasez, el uso desmedido y la contaminación. Debido a esto, es necesario replantear su modelo de gestión con el fin de reducir el impacto de estas situaciones [1] y así encaminarnos a una nueva revolución del agua [2]. En vista de lo anterior, algunos estudios sobre agua potable han tenido en cuenta aspectos tales como la integración de metodologías que involucran tanto aspectos ambientales como económicos en el sur de Florida, Estados Unidos [3], o la proposición de modelos para integrar y optimizar las infraestructuras de agua urbana en Ulsan, Corea del Sur [4].

La complejidad de los sistemas de abastecimiento de agua permite entonces la aplicación de métodos de evaluación ambiental con un alcance más amplio, como el Análisis del Ciclo de Vida (ACV). Para Comas y Morera [5], el número de trabajos sobre sistemas de abastecimiento de agua evaluados mediante ACV ha aumentado en los últimos años debido a la necesidad de comparar sistemas de tratamiento de agua tradicionales con sistemas alternativos. Lundie et al. [6] presentaron un completo ACV para el uso doméstico del agua en el sistema de abastecimiento de Sidney, Australia; De Haas et al. [7] ofrecieron una comparación entre dos escenarios en Gold Coast, Australia para el suministro de agua potable por medio del ACV; Stokes y Horvath [8] propusieron un análisis comparativo de los sistemas urbanos de abastecimiento de agua para los condados de Marin y San Diego en California, USA con base en ACV.

Lassaux et al. [9] utilizaron la metodología del ACV para estudiar los impactos ambientales del sistema de agua urbana en la región de Valonia (Bélgica), en cinco escenarios. Friedrich et al. [10] utilizaron ACV para analizar la gestión del agua urbana en el municipio de eThekwini (donde se encuentra la ciudad de Durban) en Sudáfrica. Mohamed et al. [11] compararon la situación actual con escenarios hipotéticos por medio de ACV para el caso de Alejandría, Egipto. También, Amores et al. [12] estudiaron el ciclo urbano del agua en Tarragona, España a partir de la comparación de tres escenarios: situación actual, reciclado del agua y escasez. Barjoveanu et al. [13] analizaron el sistema de abastecimiento de agua de Iasi, Rumania, mientras que en Portugal, Lemos et al. [14] estudiaron el sistema de abastecimiento de agua de la ciudad de Aveiro.

Los trabajos mencionados implican que, en la medida en que el uso del agua se dé en el marco de un sistema coherente, desde la captación hasta la devolución, el consumo del agua será más eficaz [1], se podrá abastecer agua de buena calidad y se conservarán las funciones biológicas, hidrológicas y químicas de los ecosistemas [15]. En pos de contribuir con esta visión desde el contexto colombiano, este manuscrito 
presenta los resultados del primer ACV para el sistema de suministro de agua de la ciudad de Pereira, Colombia, enfocado en el agua potable para uso doméstico. El estudio se realizó con el fin de establecer los impactos ambientales, tanto del ciclo de vida de dicho sistema, como de algunos escenarios hipotéticos. Para lograrlo, se propuso y se analizó un escenario base. Este escenario estuvo compuesto por tres elementos: 1) ciclo de vida del agua potable para uso doméstico y sus características, 2) inventario de insumos y flujos de materia y energía del ciclo e impactos ambientales, tanto de punto medio como de punto final y 3 ) evaluación de los impactos ambientales de punto medio y de punto final.

Una vez realizado el ACV para el escenario base, se propusieron tres escenarios ambientales hipotéticos: i) reducción del $20 \%$ del consumo de agua potable, ii) tratamiento primario avanzado de aguas residuales, y iii) combinación de los escenarios i y ii. Los impactos ambientales se evaluaron utilizando el método Impact 2002+, el cual se basa en un enfoque combinado de punto medio y daño que permite evaluar impactos ambientales, tanto de punto medio, como de punto final (o de daño). Lo anterior es posible debido a que Impact 2002+ permite vincular los resultados del ICV (Inventario de Ciclo de Vida) mediante categorías de punto medio, a unas categorías de punto final o categorías de daño [16], asimismo, este método tiene en cuenta varias categorías de impacto relacionadas con el uso del agua.

La evaluación de impactos permitió comparar dos etapas en el ciclo de vida: la primera es la que arroja como producto el agua potable y comprende las actividades de conservación de cuencas y recolección de agua cruda, tratamiento del agua cruda y distribución de agua potable. La segunda tiene como producto el agua residual y comprende las fases de uso doméstico y recolección, transporte y disposición de aguas residuales.

\section{MATERIALES Y MÉTODOS}

\subsection{Metodología}

Este trabajo se hizo en cuatro etapas. Las primeras tres etapas se realizaron con base en el análisis de ciclo de vida (ACV en adelante), mientras que la cuarta etapa se hizo como complemento de los resultados de las tres primeras. Se empleó el marco general del ACV (Normas ISO 14040 y 14044) para identificar el impacto ambiental asociado a los procesos de producción, uso y disposición final del agua potable para uso doméstico.

El ACV es una metodología para la evaluación del desempeño ambiental que permite identificar los aspectos e impactos ambientales asociados a un producto o servicio. Todo ACV debe cumplir con las siguientes etapas: i) definición del objetivo y alcance del estudio, la cual permite definir la unidad funcional y los 
límites del sistema para la descripción del ciclo de vida; ii) recopilación de insumos y productos del sistema (Inventario del Ciclo de Vida, ICV); iii) evaluación del impacto del ciclo de vida, EICV; y iv) interpretación de los resultados del ICV y EICV junto con la evaluación del impacto en relación con los objetivos del estudio en cuestión [17-18].

\subsubsection{Descripción del ciclo de vida del agua potable para uso doméstico}

Pereira está ubicada al oeste de Colombia y es la capital del departamento de Risaralda. Toda el agua potable utilizada en Pereira proviene del río Otún, cuyo nacimiento se encuentra a 3.900 metros sobre el nivel del mar, en el Parque Nacional Natural Los Nevados. La empresa Aguas \& Aguas es responsable del servicio de suministro de agua para uso doméstico, comercial e industrial. Según datos de la empresa, en 2017 había un total de 147.754 usuarios suscritos, de los cuales el $91,4 \%$ eran residenciales. En ese año los hogares consumieron 18.858.434 metros cúbicos de agua, que representan el $81 \%$ del volumen total consumido en la ciudad.

De la mano de la metodología del ACV se definió como objetivo para este trabajo la identificación de los impactos ambientales relacionados con la producción, uso y disposición final del agua potable para uso doméstico en Pereira, Colombia. Así mismo, se definió como unidad funcional $1 \mathrm{~m}^{3}$ de agua potable para uso doméstico. Una vez definidos el objetivo y alcance del ACV, se estableció que el volumen total de agua consumida por los usuarios residenciales durante el $2017\left(18.858 .434 \mathrm{~m}^{3}\right)$ sería el flujo de referencia para el sistema. La figura 1 permite apreciar las etapas del ciclo de vida y las entradas y salidas de materiales y energía.

Con base en la definición del objetivo y el alcance del estudio, se estableció que el ciclo de vida del agua potable para uso doméstico en Pereira consta de cinco etapas. La primera etapa es la conservación de la cuenca del río Otún y la captación de agua cruda, para ello es necesaria la gestión ambiental de la cuenca y la obtención de agua del río. En la segunda etapa se produce el tratamiento o purificación del agua cruda. En las primeras etapas de este proceso el agua se trata químicamente, se añaden cal hidratada y carbón activado para mejorar la alcalinidad y eliminar las partículas orgánicas en suspensión.

Este tratamiento preliminar termina con un proceso llamado filtración de arena, el cual consiste en remover grava, arena y material particulado. Posteriormente, se lleva el agua a las tres plantas de tratamiento de la empresa (Quimbaya, Písamo y La Aurora). En cada una de ellas se llevan a cabo los siguientes procesos para completar el tratamiento: coagulación, floculación, sedimentación, filtración, desinfección y ajuste de $\mathrm{pH}$. 

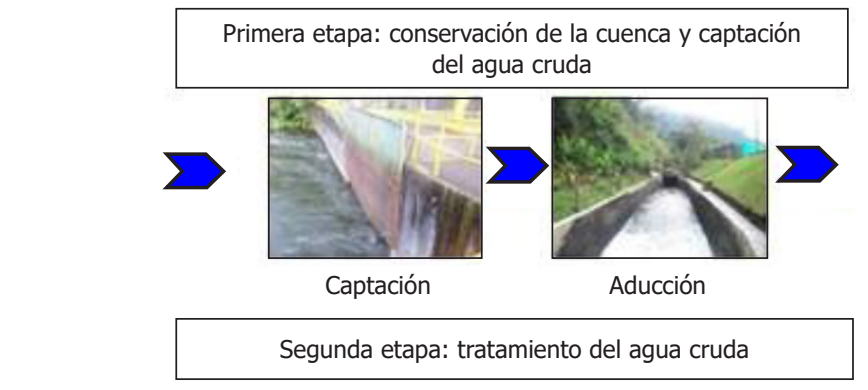

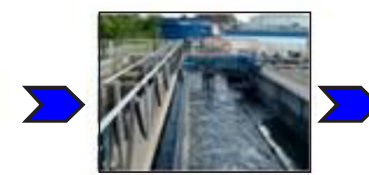

Pre-tratamiento

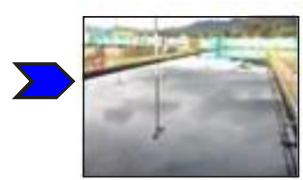

Sedimentación

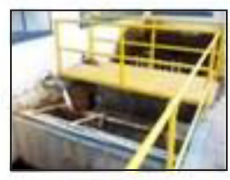

Coagulación

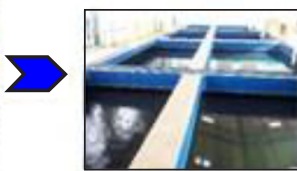

Filtración

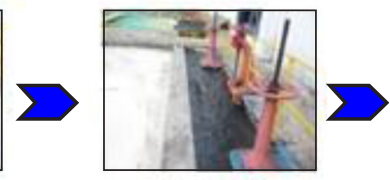

Floculación

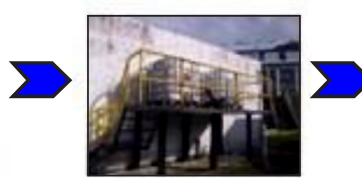

Ajuste de $\mathrm{pH}$

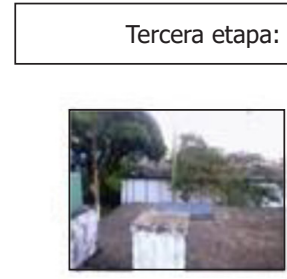

Almacenamiento

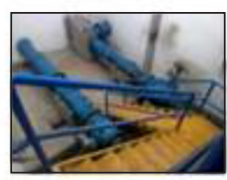

Distribución

Cuarta etapa: uso doméstico

Quinta etapa: recolección, transporte y disposición del agua residual

Figura 1. Etapas del ciclo de vida del agua potable para uso doméstico en Pereira, Colombia

Fuente: elaboración propia

La tercera etapa es la distribución del agua potable a través de la red principal. La longitud de la red de distribución es de aproximadamente $761 \mathrm{~km}$. La distribución de agua potable se realiza por medio de tuberías con diámetros que varían entre $1 \mathrm{y}$ 40 pulgadas. La cuarta etapa comienza cuando el agua llega a los usuarios (para este estudio solo se consideraron los consumidores residenciales). Esta etapa se denomina uso doméstico de agua y comprende, en términos generales, las siguientes actividades: cocción de alimentos, cuidado personal, limpieza y tareas domésticas, lavandería, riego y jardinería. 
Por último, durante la quinta etapa (recolección, transporte y disposición de aguas residuales) las aguas residuales entran al sistema de alcantarillado. Según los datos proporcionados por la empresa, la red de alcantarillado tiene una extensión de $670 \mathrm{~km}$, de los cuales $65 \mathrm{~km}$ están destinados a colectores e interceptores.

\subsubsection{Inventario del ciclo de vida del agua potable para uso doméstico}

Una vez definidos el objetivo y el alcance del trabajo, se recopilaron datos de entradas y salidas de materia y energía (electricidad, agua, insumos químicos, pérdidas de agua, etc.) suministrados por la compañía para una operación anual (ver figura 2). También se estimaron otros datos importantes como: transporte, excavaciones de redes y emisiones de elementos o compuestos químicos distintos al aluminio. Una vez obtenidos los datos se les relacionó con los procesos del ciclo, así como con la unidad funcional.

Según la información suministrada por Aguas \& Aguas, se determinó que durante la operación anual se requiere la entrada de $1,54 \mathrm{~m}^{3}$ de agua cruda para generar la unidad funcional $\left(1 \mathrm{~m}^{3}\right)$ como agua potable para uso doméstico. Por consiguiente, se presenta una pérdida de $0,54 \mathrm{~m}^{3}$ a lo largo de los procesos anteriores. Según la compañía, dichas pérdidas de agua se deben a factores tales como el uso de agua potable en la limpieza de tanques y otras instalaciones, consumo interno de la empresa, errores de facturación, conexiones no autorizadas al sistema de suministro de agua y fugas causadas por rupturas de tuberías.

El ICV se realizó en consonancia con la norma ISO 14044 para compilar, validar datos y posteriormente determinar su correlación con los procesos unitarios y la unidad funcional determinada. El resultado del ICV es un inventario con entradas y salidas de materia y energía en todo el sistema. En este trabajo, los datos fueron recogidos, tanto por medio de entrevistas con el personal de Aguas \& Aguas, como de revisiones de documentos de la empresa sobre aspectos clave para una operación anual típica.

\subsubsection{Evaluación de los impactos ambientales del ciclo de vida}

La evaluación de los impactos ambientales del ciclo de vida se hizo mediante la Evaluación de Impacto de Ciclo de Vida (EICV). Esta se lleva a cabo sobre la base de un inventario anterior. La EICV permite validar la coherencia del objetivo y alcance del estudio con respecto a los datos y resultados del ACV. La EICV presentada en este artículo se realizó con la aplicación del método para evaluación de impactos ambientales denominado Impact 2002+, herramienta desarrollada por la Ecole Polytechnique Fédérale de Lausanne (EPFL) en Suiza. La tabla 1 muestra las 15 categorías de punto medio analizadas para este estudio, así como sus categorías correlativas de punto final. 


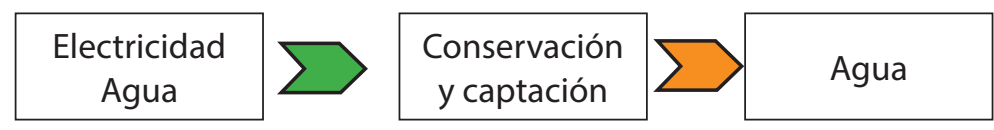

\begin{tabular}{|c|}
\hline Electricidad \\
Insumos \\
químicos \\
(Carbón activado, \\
sulfato de \\
aluminio sólido, \\
sulfato de \\
aluminio líquido, \\
cloro líquido, \\
polivinilo de \\
aluminio, soda \\
cáustica) \\
Transporte \\
\hline
\end{tabular}

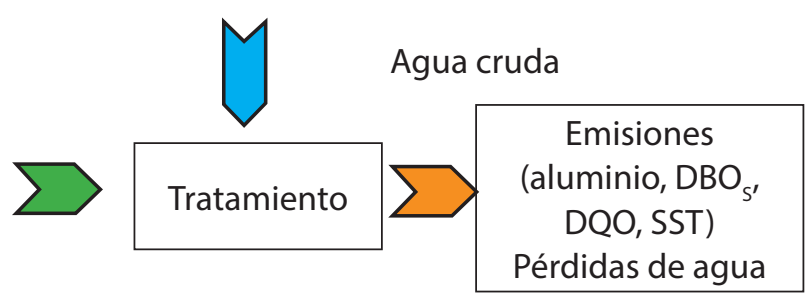

\begin{tabular}{|c|}
\hline Electricidad \\
Agua \\
Tubería de PVC \\
Transporte \\
Excavadora \\
hidráulica \\
(excavación) \\
\hline
\end{tabular}
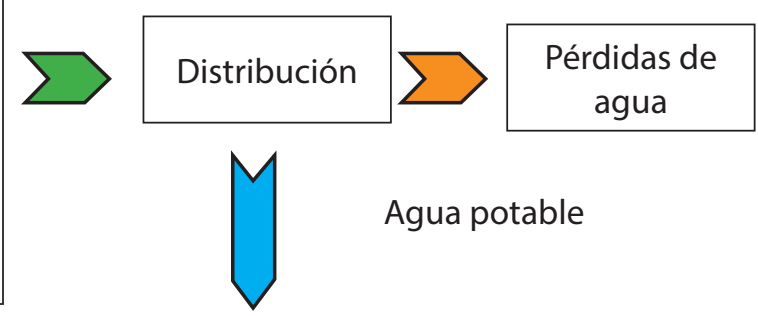

Agua potable

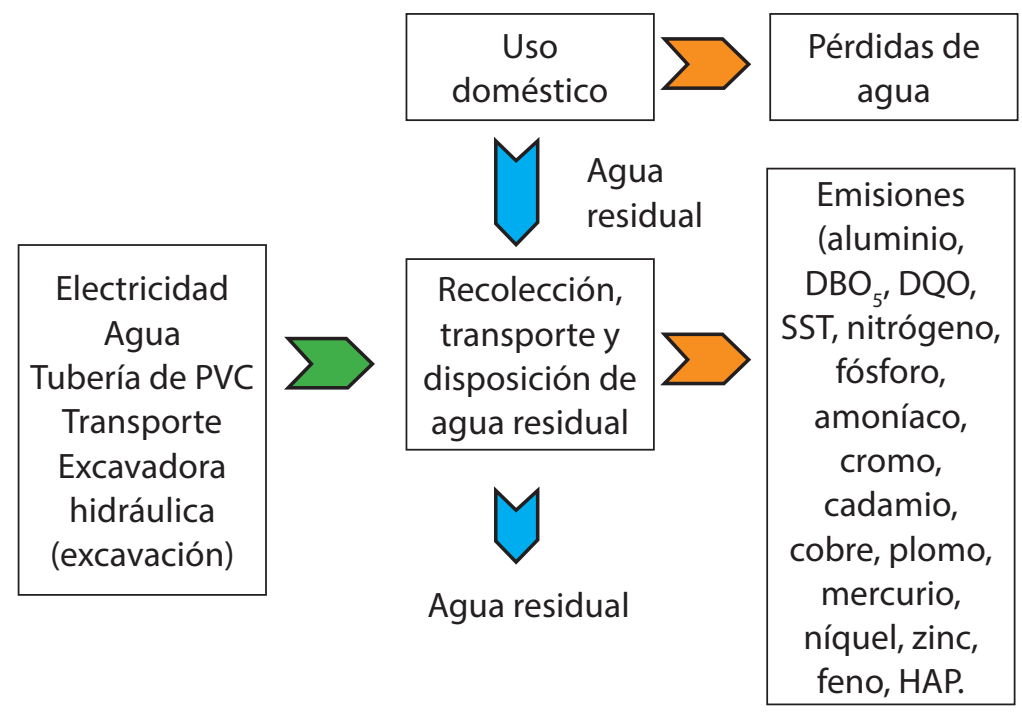

Figura 2. Flujos de materia y energía del ciclo de vida del agua potable para uso doméstico en Pereira Fuente: elaboración propia 
Tabla 1. Categorías de punto medio analizadas y sus categorías correlativas de punto final

\begin{tabular}{|c|c|}
\hline Categoría de punto medio & Categoria de punto final \\
\hline Toxicidad humana (efectos cancerígenos) & \multirow{5}{*}{ Salud humana } \\
\hline Toxicidad humana (efectos no cancerígenos) & \\
\hline Efectos respiratorios (por sustancias inorgánicas) & \\
\hline Radiación ionizante & \\
\hline Deterioro de la capa de ozono & \\
\hline \multirow{2}{*}{ Efectos respiratorios por sustancias orgánicas (oxidación fotoquímica) } & Salud humana \\
\hline & Calidad de los ecosistemas \\
\hline \multicolumn{2}{|l|}{ Toxicidad acuática } \\
\hline \multicolumn{2}{|l|}{ Toxicidad terrestre } \\
\hline \multicolumn{2}{|l|}{ Acidificación por uso del suelo/eutrofización } \\
\hline Acidificación acuática & \multirow[t]{3}{*}{ Calidad de los ecosistemas } \\
\hline Eutrofización acuática & \\
\hline Ocupación del suelo & \\
\hline Calentamiento global & Cambio climático \\
\hline Energía no renovable & \multirow{2}{*}{ Recursos } \\
\hline Extracción de minerales & \\
\hline
\end{tabular}

Fuente: elaboración propia

Se utilizó la base de datos Ecoinvent 3.0 y el software SimaPro 8.0.2 para la clasificación, caracterización y normalización de impactos. La caracterización facilita el conocimiento de los impactos de punto medio, mientras que la normalización permite una aproximación a la magnitud de los impactos ambientales de punto final.

\subsubsection{Definición de escenarios ambientales hipotéticos del ciclo de vida del agua potable para uso doméstico}

Este último paso tiene en cuenta la identificación de cuestiones importantes basadas en los resultados de ICV y EICV y su interpretación. Para la discusión, aparte del escenario base, en este trabajo se proponen tres escenarios ambientales hipotéticos. Cabe señalar que, para obtener los impactos totales tanto de punto medio como de punto final en todos los escenarios, los resultados iniciales (por $1 \mathrm{~m}^{3}$ ) se multiplicaron por el flujo de referencia correspondiente. En el escenario base y en el escenario hipotético 2 se utilizó el flujo de referencia del $2017\left(18.858 .434 \mathrm{~m}^{3}\right)$, mientras que en los escenarios hipotéticos 1 y 3 se realizaron cálculos con un $80 \%$ de ese flujo $\left(15.086 .747 \mathrm{~m}^{3}\right)$. Tanto 
el escenario hipotético 1 como el 3 se basan en considerar una reducción del consumo del $20 \%$ en relación con el volumen total consumido en el 2017. En ambos escenarios (1 y 3 ) se supone que tanto los insumos como los flujos de aguas residuales se presentan en cantidades iguales a las de un año típico. Así mismo, la unidad funcional permanece igual $\left(1 \mathrm{~m}^{3}\right)$, lo que cambia es el flujo de referencia, que se supone que también se reducirá en un $20 \%$.

Por otra parte, se analiza un escenario que reduce la contaminación orgánica del agua residual mediante tratamiento primario avanzado (escenario 2). La formulación de este escenario se basa en dos hipótesis: i) el tratamiento propuesto permite las siguientes remociones de contaminación orgánica: $\mathrm{DBO}_{5} 55 \%$, DQO $45 \%$, SST $70 \%$, fósforo $75 \%$ y nitrógeno $30 \%$ [19]; y (ii) se utilizarían $0,56 \mathrm{kWh}$ de energía eléctrica por $\mathrm{m}^{3}$ de agua tratada [14]. Dicho tratamiento se eligió como referencia para este estudio porque es el sistema que en el futuro está destinado a emplearse para el manejo de aguas residuales en la ciudad de Pereira. En este escenario, el flujo de referencia es igual al del escenario de referencia.

Finalmente, se analiza un tercer escenario (escenario 3) que considera una combinación de los dos primeros escenarios, bajo el supuesto de que ambos se desarrollan de forma simultánea.

\section{RESULTADOS Y DISCUSIÓN}

\subsection{Inventario de Ciclo de vida}

En la tabla 2 se presentan los resultados del Inventario de Ciclo de Vida del agua potable para uso doméstico en la ciudad de Pereira en el año 2017.

Tabla 2. Inventario de Ciclo de Vida. Unidad funcional: $1 \mathrm{~m}^{3}$ de agua potable para uso doméstico

\begin{tabular}{|c|c|c|c|c|c|}
\hline \multicolumn{6}{|c|}{ Primera etapa: conservación de la cuenca y captación del agua cruda } \\
\hline Entradas & Indicador & Unidad & Emisiones al agua & Indicador & Unidad \\
\hline Agua cruda & 1,5 & $\mathrm{~m}^{3}$ & Agua & $1,4 \mathrm{E}-5$ & $\mathrm{~m}^{3}$ \\
\hline Electricidad & 7,E-4 & $\mathrm{kWh}$ & & & \\
\hline Agua & $1,4 \mathrm{E}-5$ & $\mathrm{~m}^{3}$ & & & \\
\hline \multicolumn{6}{|c|}{ Segunda etapa: tratamiento } \\
\hline Entradas & Indicador & Unidad & Emisiones al agua & Indicador & Unidad \\
\hline Agua cruda & 1,5 & $\mathrm{~m}^{3}$ & Aluminio & $1,4 \mathrm{E}-4$ & $\mathrm{~kg}$ \\
\hline Cal hidratada & $6,2 \mathrm{E}-4$ & $\mathrm{~kg}$ & $\mathrm{DBO}_{5}$ & $2,4 \mathrm{E}-6$ & $\mathrm{~kg}$ \\
\hline Carbón activado & $8,8 \mathrm{E}-4$ & $\mathrm{~kg}$ & DQO & $6,4 \mathrm{E}-6$ & $\mathrm{~kg}$ \\
\hline Sulfato de aluminio sólido & $7,2 \mathrm{E}-3$ & $\mathrm{~kg}$ & & & \\
\hline
\end{tabular}




\begin{tabular}{|c|c|c|c|c|c|}
\hline \multicolumn{6}{|c|}{ Segunda etapa: tratamiento } \\
\hline Entradas & Indicador & Unidad & Emisiones al agua & Indicador & Unidad \\
\hline Sulfato de aluminio líquido & 1,1E-2 & $\mathrm{kg}$ & SST & 4,8E-6 & $\mathrm{kg}$ \\
\hline Cloro líquido & $2,4 \mathrm{E}-3$ & $\mathrm{~kg}$ & Pérdidas de agua & $1,1 \mathrm{E}-1$ & $\mathrm{~m}^{3}$ \\
\hline Polivinilo de aluminio & $3,7 \mathrm{E}-3$ & $\mathrm{~kg}$ & & & \\
\hline Soda cáustica & $1,2 \mathrm{E}-3$ & $\mathrm{~kg}$ & & & \\
\hline Electricidad & $3,0 \mathrm{E}-2$ & $\mathrm{kWh}$ & & & \\
\hline Transporte de químicos & $2,1 \mathrm{E}-1$ & $\mathrm{tkm}$ & & & \\
\hline \multicolumn{6}{|c|}{ Tercera etapa: distribución } \\
\hline Entradas & Indicador & Unidad & Emisiones al agua & Indicador & Unidad \\
\hline Agua tratada & 1,4 & $\mathrm{~m}^{3}$ & Pérdidas de agua & 4,3E-1 & $\mathrm{m}^{3}$ \\
\hline Tuberías de PVC & $2,4 \mathrm{E}-3$ & $\mathrm{~kg}$ & & & \\
\hline Transporte de tuberías & $6,4 \mathrm{E}-2$ & $\mathrm{tkm}$ & & & \\
\hline Excavadora hidráulica (Excavación) & $2,8 \mathrm{E}-4$ & $\mathrm{~m}^{3}$ & & & \\
\hline Electricidad & $7,0 \mathrm{E}-4$ & $\mathrm{kWh}$ & & & \\
\hline Agua potable & $8,8 \mathrm{E}-5$ & $\mathrm{~m}^{3}$ & & & \\
\hline \multicolumn{6}{|c|}{ Cuarta etapa: uso doméstico } \\
\hline Entradas & Indicador & Unidad & Emisiones al agua & Indicador & Unidad \\
\hline Agua distribuida & 1 & $\mathrm{~m}^{3}$ & Pérdidas de agua & 0,2 & $\mathrm{~m}^{3}$ \\
\hline \multicolumn{6}{|c|}{ Quinta etapa: recolección, transporte y disposición de aguas residuales } \\
\hline Entradas & Indicador & Unidad & Emisiones al agua & Indicador & Unidad \\
\hline Agua potable & $1,6 \mathrm{E}-6$ & $\mathrm{~m}^{3}$ & Agua residual & 0,8 & $\mathrm{~m}^{3}$ \\
\hline Tubería de PVC & $2,4 \mathrm{E}-4$ & $\mathrm{~kg}$ & $\mathrm{DBO}_{5}$ & 2,1E-1 & $\mathrm{kg}$ \\
\hline Excavadora hidráulica (excavación) & $3,0 \mathrm{E}-5$ & $\mathrm{~m}^{3}$ & DQO & $2,6 \mathrm{E}-1$ & $\mathrm{~kg}$ \\
\hline \multirow[t]{14}{*}{ Electricidad } & $2,8 \mathrm{E}-4$ & $\mathrm{kWh}$ & SST & $0,1 \mathrm{E}-1$ & $\mathrm{~kg}$ \\
\hline & & & Nitrógeno & $1,6 \mathrm{E}-1$ & $\mathrm{~kg}$ \\
\hline & & & Fósforo & $3,2 \mathrm{E}-4$ & $\mathrm{~kg}$ \\
\hline & & & Amoníaco & $1,1 \mathrm{E}-2$ & $\mathrm{~kg}$ \\
\hline & & & Cromo & $1,1 \mathrm{E}-5$ & $\mathrm{~kg}$ \\
\hline & & & Cadmio & $9,6 \mathrm{E}-7$ & $\mathrm{~kg}$ \\
\hline & & & Cobre & $3,2 \mathrm{E}-5$ & $\mathrm{~kg}$ \\
\hline & & & Plomo & $2,6 \mathrm{E}-5$ & $\mathrm{~kg}$ \\
\hline & & & Mercurio & $9,6 \mathrm{E}-7$ & $\mathrm{~kg}$ \\
\hline & & & Níquel & $1,1 \mathrm{E}-5$ & $\mathrm{~kg}$ \\
\hline & & & Zinc & $9,6 \mathrm{E}-6$ & $\mathrm{~kg}$ \\
\hline & & & Fenol & $1,9 \mathrm{E}-5$ & $\mathrm{~kg}$ \\
\hline & & & HAP (PAH) & $6,4 \mathrm{E}-4$ & $\mathrm{~kg}$ \\
\hline & & & Cloroformo & $1,9 \mathrm{E}-5$ & $\mathrm{~kg}$ \\
\hline
\end{tabular}

$\mathrm{DBO}_{5}$ : Demanda bioquímica de oxígeno; DQO: Demanda química de oxígeno; SST: Sólidos suspendidos totales HAP: Hidrocarburos aromáticos policíclicos 
El tratamiento del agua cruda es la etapa del ciclo de vida que mayor consumo de electricidad presenta $\left(0,03 \mathrm{kWh} / \mathrm{m}^{3}\right)$ debido al uso de energía eléctrica para procesos tales como la adición de sustancias químicas que facilitan la potabilización. Barjoveanu et al. [13], Friedrich et al. [10] y Lundie et al. [6] obtuvieron resultados de consumo por metro cúbico similares $\left(0,04 \mathrm{kWh} / \mathrm{m}^{3}, 0,09 \mathrm{kWh} / \mathrm{m}^{3}\right.$ y $0,08 \mathrm{kWh} / \mathrm{m}^{3}$ respectivamente). Con relación a las pérdidas de agua, esta es mayor en la etapa de distribución $\left(0,43 \mathrm{~m}^{3}\right)$ a causa de los factores reseñados en la descripción del inventario de ciclo de vida (numeral 1.1.2). Este resultado es similar a los obtenidos por Lassaux et al. [9] $\left(0,42 \mathrm{~m}^{3}\right)$, Friedrich et al. [10] $\left(0,42 \mathrm{~m}^{3}\right)$ y Slagstad y Brattebø [21] $\left(0,47 \mathrm{~m}^{3}\right)$ para la etapa de distribución.

\subsection{Impactos ambientales del ciclo de vida: escenario de referencia}

Con respecto a los impactos ambientales establecidos, se debe aclarar que la discusión de resultados presenta limitaciones debido a que a la fecha de elaboración del presente artículo no se encontraron estudios similares (es decir, estudios en los que se analice el ciclo de vida de todo el sistema de suministro del agua potable) que se hubiesen realizado con base en la metodología Impact 2002+. Por lo tanto, es difícil llevar a cabo comparaciones precisas entre este y otros trabajos.

\subsubsection{Impactos ambientales de punto medio}

La tabla 3 muestra los resultados de los impactos ambientales del agua potable para uso doméstico en 15 categorías de punto medio. Cabe destacar que el conjunto de procesos relacionados con la producción de agua potable fue responsable de la mayoría de los impactos de punto medio (entre el 75 y el $100 \%$ en 12 de las 15 categorías). Los procesos relacionados con el agua potable generaron también la mayor parte de los impactos de punto medio para la mayoría de categorías en los trabajos de Lemos et al. [14] (entre 39 y $45 \%$ ), Barjoveanu et al. [13] (entre 70 y 78 \%) y Amores et al. [12] (entre 21 y $37 \%$ ).

El conjunto de actividades relacionadas con las aguas residuales (uso doméstico de agua potable y recolección, transporte y disposición final de aguas residuales) tuvo la mayor participación en tres categorías (ver tabla 3), siendo responsable de la mayor parte de dichos impactos (entre 96 y $100 \%$ ). En este sentido, Mohamed et al. [11], Barjoveanu et al. [13] y Friedrich et al. [10], encontraron que las aguas residuales no tratadas causan eutrofización debido a la presencia de nutrientes en estas. 
Tabla 3. Impactos ambientales de punto medio del agua potable para uso doméstico en Pereira en el 2017

\begin{tabular}{|c|c|c|c|c|}
\hline Categoría de impacto & Unidad & Total & $\begin{array}{c}\text { Agua } \\
\text { potable } \\
(\%)\end{array}$ & $\begin{array}{c}\text { Agua } \\
\text { residual }^{2} \\
(\%)\end{array}$ \\
\hline Efectos cancerígenos & $\mathrm{kg} \mathrm{C}_{2} \mathrm{H}_{3} \mathrm{Cl}$ eq & $2,9 \mathrm{E}+06$ & 3,7 & 96,3 \\
\hline Efectos no cancerígenos & $\mathrm{kg} \mathrm{C}_{2} \mathrm{H}_{3} \mathrm{Cl}$ eq & $5,7 \mathrm{E}+05$ & 81,4 & 18,6 \\
\hline $\begin{array}{l}\text { Efectos respiratorios (sustancias } \\
\text { inorgánicas) }\end{array}$ & $\mathrm{kg}$ PM 2,5 eq & $4,3 \mathrm{E}+03$ & 99,6 & 0,4 \\
\hline Radiación ionizante & Bq C-14 eq & $6,4 \mathrm{E}+07$ & 99,9 & 0,1 \\
\hline Deterioro de la capa de ozono & kg CFC-11 eq & $6,2 \mathrm{E}-01$ & 100,0 & 0,0 \\
\hline $\begin{array}{l}\text { Efectos respiratorios (sustancias } \\
\text { orgánicas) }\end{array}$ & $\mathrm{kg} \mathrm{C}_{2} \mathrm{H}_{4}$ eq & $2,4 \mathrm{E}+03$ & 99,9 & 0,1 \\
\hline Ecotoxicidad acuática & kg TEG agua & $1,9 \mathrm{E}+10$ & 75,4 & 24,6 \\
\hline Toxicidad terrestre & kg TEG suelo & $1,5 \mathrm{E}+08$ & 100,0 & 0,0 \\
\hline Acidificación del suelo / eutrofización & $\mathrm{kg} \mathrm{SO}_{2} \mathrm{eq}$ & $1,2 \mathrm{E}+05$ & 99,7 & 0,3 \\
\hline Acidificación acuática & $\mathrm{kg} \mathrm{SO}_{2} \mathrm{eq}$ & $6,9 \mathrm{E}+04$ & 99,9 & 0,1 \\
\hline Eutrofización acuática & $\mathrm{kg} \mathrm{PO} \mathrm{P}_{4} \mathrm{P}-\mathrm{lim}$ & $6,2 \mathrm{E}+05$ & 3,6 & 96,4 \\
\hline Ocupación del suelo & m2 org. Suelo arable & $1,9 \mathrm{E}+05$ & 0,2 & 99,8 \\
\hline Calentamiento global & $\mathrm{kg} \mathrm{CO}_{2} \mathrm{eq}$ & $3,7 \mathrm{E}+06$ & 99,4 & 0,6 \\
\hline Energía no renovable & MJ energía primaria & $6,4 \mathrm{E}+07$ & 99,2 & 0,8 \\
\hline Extracción de minerales & MJ energía excedente & $5,0 \mathrm{E}-03$ & 99,9 & 0,1 \\
\hline
\end{tabular}

${ }^{1}$ Este conjunto abarca los procesos de capacitación, tratamiento y distribución del agua potable.

${ }^{2}$ Este conjunto comprende procesos de uso doméstico de agua potable y eliminación de aguas residuales.

Fuente: elaboración propia

\subsubsection{Impactos ambientales de punto final}

Tal como se muestra en la tabla 4, el mayor daño se produce en la categoría de salud humana con el 66,3\%. Los procesos relacionados con el agua potable generaron el mayor impacto de punto final (entre 90 y $99 \%$ ) en tres categorías (calidad de los ecosistemas, cambio climático y recursos), mientras que los procesos relacionados con aguas residuales tuvieron el mayor impacto en la salud humana (65\%).

Por último, los procesos e insumos químicos relacionados con la producción y distribución de agua potable contribuyeron con el 56,1 \% del daño final total. Por su parte, las actividades relacionadas con las aguas residuales contribuyeron con el 
restante 43,9\%. Con respecto a esta última cuestión, Lemos et al. [14] llegaron a una conclusión similar, sin embargo, Barjoveanu et al. [13] encontraron que la gestión de aguas residuales generó más impacto que el conjunto de procesos relacionados con el agua potable. Esto se puede deber a las diferentes metodologías de evaluación de punto final que utilizaron, ya que los primeros se basaron en el método ReCiPe, mientras que los segundos emplearon una combinación de las metodologías CML y Ecoscarcity 2006.

Tabla 4. Impactos ambientales de punto final del agua potable para uso doméstico en Pereira durante el 2017

\begin{tabular}{|l|c|c|c|c|}
\hline \multicolumn{1}{|c|}{ Categoría de daño } & $\begin{array}{c}\text { Total } \\
\text { puntos }\end{array}$ & $\%$ & $\begin{array}{c}\text { Agua potable }^{1} \\
\text { (\%) }\end{array}$ & $\begin{array}{c}\text { Agua residual }^{2} \\
\text { (\%) }\end{array}$ \\
\hline Salud humana & $1,4 \mathrm{E}+03$ & 66,3 & 35,0 & 65,0 \\
\hline Calidad de los ecosistemas & $1,3 \mathrm{E}+02$ & 6,0 & 89,9 & 10,1 \\
\hline Cambio climático & $2,8 \mathrm{E}+02$ & 13,0 & 99,4 & 0,6 \\
\hline Recursos naturales & $3,2 \mathrm{E}+02$ & 14,7 & 99,2 & 0,8 \\
\hline Daño total punto final & $2,2 \mathrm{E}+03$ & 100 & 56,1 & 43,9 \\
\hline
\end{tabular}

${ }^{1}$ Este conjunto abarca los procesos de recolección, tratamiento y distribución del agua potable.

${ }^{2}$ Este conjunto comprende procesos de uso doméstico de agua potable y eliminación de aguas residuales.

Fuente: elaboración propia

\subsection{Evaluación del impacto de ciclo de vida: escenarios ambientales hipotéticos}

\subsubsection{Impactos ambientales de punto medio}

Con el fin de analizar la sensibilidad del modelo propuesto en el ACV, se evaluaron tres escenarios ambientales hipotéticos. Para el primer escenario se estimó una reducción del $20 \%$ de los impactos en todas las categorías de punto medio (ver figura 3), en relación con el escenario de línea de base.

Al considerar el segundo escenario, la figura 3 muestra que 8 categorías reportarían una reducción de impactos (entre 1 y $60 \%$ ), mientras que las 7 restantes mostrarían un incremento en impactos negativos (entre 0 y $73 \%$ ). Lo anterior concuerda con lo establecido por Barjoveanu et al. [13], quienes encontraron mediante el método CML que el mejoramiento del tratamiento del agua residual puede reducir hasta en $13 \%$ el impacto en la categoría de eutrofización y aumentar el impacto de otras categorías. Si se produjera el escenario número 3, se daría una reducción de los impactos de punto medio en 11 categorías en lugar de 8 . Esta reducción variaría entre 11 y $68 \%$. 
Por otro lado, el escenario 3 implicaría que el número de categorías con incrementos en sus impactos de punto medio disminuya de 7 a 4 . Estos aumentos estarían entre 17 y $38 \%$.

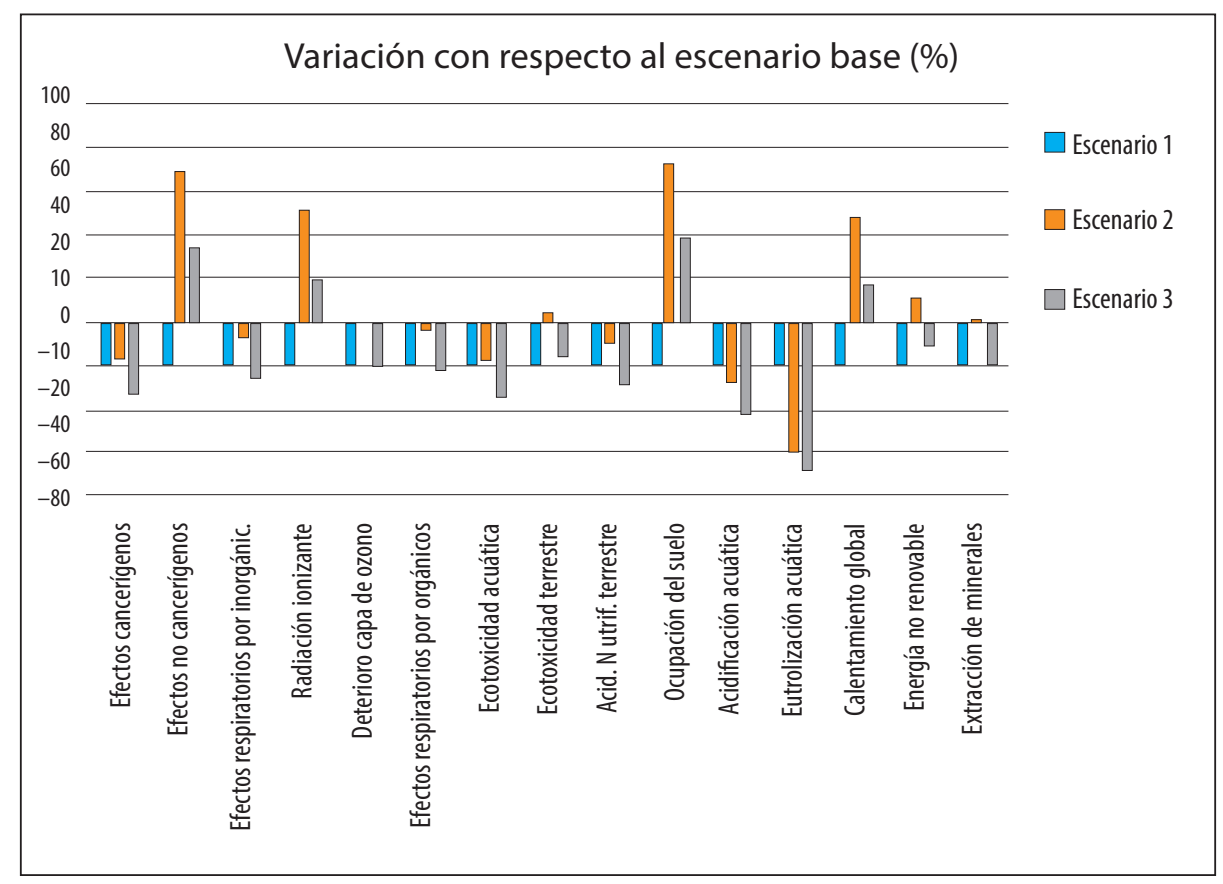

Figura 3. Impactos ambientales de punto medio para escenarios hipotéticos

Fuente: elaboración propia

\subsubsection{Impactos ambientales de punto final}

Tal como se muestra en la figura 4, reducir el consumo de agua en un $20 \%$ (escenario 1) también implicaría una reducción del $20 \%$, tanto en los impactos de todas las categorías como en el daño total. El tratamiento de aguas residuales por sí solo (escenario 2) generaría aumentos en los impactos en las cuatro categorías (entre 22 y $96 \%$ ), mientras que el daño total incrementaría su impacto en un $36 \%$. Se debe tener en cuenta que este resultado depende del límite analizado. Si los escenarios 1 y 2 ocurrieran simultáneamente (escenario 3), los impactos aumentarían entre 2 y $56 \%$ en tres categorías (con excepción de salud humana). Por su parte, el daño total aumentaría un $8 \%$. 


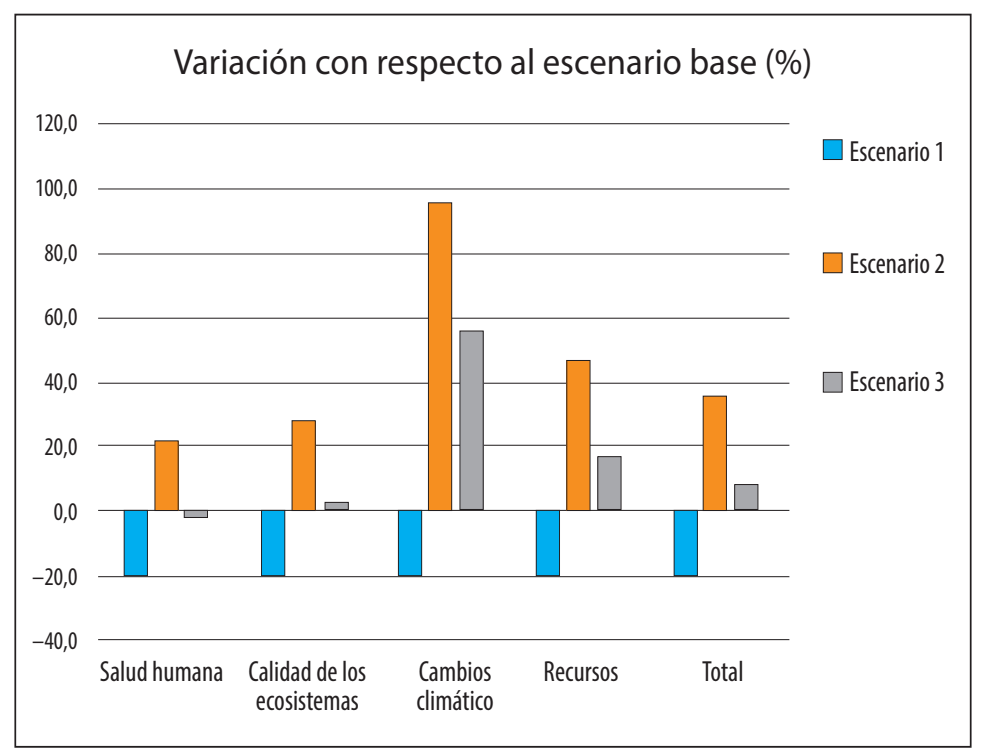

Figura 4. Impactos ambientales de punto final para escenarios hipotéticos

Fuente: elaboración propia

\section{CONCLUSIONES}

Se realizó un ACV del agua potable para uso doméstico en Pereira cuyo enfoque se centró en las etapas de la metodología de $\mathrm{ACV}$ y se tomó $1 \mathrm{~m}^{3}$ de agua potable como unidad funcional para una operación anual típica. Como la ciudad no cuenta con un sistema de tratamiento de aguas residuales, los datos relacionados con los procesos asociados no se incluyeron en el escenario base. Sin embargo, el inventario resultante permite hacer una aproximación a los casos de otras ciudades con condiciones semejantes en Colombia que también carecen de tratamiento de aguas residuales. La EICV siguió el método Impact 2002+ con el objetivo de caracterizar los impactos de punto medio y normalizar los impactos de punto final. Se analizaron tres escenarios ambientales hipotéticos con respecto al agua potable para uso doméstico: el primero consideró una reducción del consumo del $20 \%$; el segundo sugirió un manejo de aguas residuales basado en el tratamiento primario avanzado; y el tercero consideró una combinación de los dos primeros escenarios.

Nuestros resultados para el escenario de referencia muestran que los procesos relacionados con la producción de agua potable fueron responsables de la mayoría de los impactos de punto medio (entre 75 y $100 \%$ ) en 12 de las 15 categorías estudiadas. Para los impactos de punto final, el agua potable también fue el principal contribuyente (56\%). Al tomar en consideración las categorías de impacto de punto medio para los 
escenarios ambientales hipotéticos, los resultados mostraron que el primer escenario conduciría a una reducción del $20 \%$ de los impactos negativos para todas las 15 categorías estudiadas. Si se implementara el segundo escenario, los impactos de punto medio se reducirían del 1 al $60 \%$ en 8 categorías, mientras que en 7 categorías los impactos aumentarían (de 0 a $73 \%$ ). La puesta en marcha del tercer escenario daría lugar a que 11 categorías vieran reducidos los impactos entre 11 y $68 \%$. Solo en 4 categorías los impactos aumentarían entre 17 y $38 \%$.

Finalmente, si se observan los resultados de punto final, se puede decir que el escenario número 1 reduciría el $20 \%$ de los impactos en cada categoría y en el daño total. La aplicación del escenario 2 significaría un aumento de los impactos en las cuatro categorías que oscila entre 22 y $96 \%$ y el daño total aumentaría en un $36 \%$. Si se produjera el escenario 3, habría incrementos en los impactos en tres categorías (entre 2 y $56 \%$ ) y en el daño total (8\%).

Con base en lo anterior, se puede decir que el tercer escenario es el más adecuado ambientalmente para el agua potable de uso doméstico en Pereira porque favorece la reducción de impactos en muchas categorías de punto medio. En cuanto a los impactos de punto final, aunque se percibirían incrementos en casi todas las categorías, estos serían menores con relación al escenario número 2. Si bien el escenario número 1 parece ser totalmente favorable, este se debe complementar con el tratamiento de aguas residuales para avanzar hacia una mejor gestión del ciclo de vida del agua para uso doméstico en Pereira.

\section{AGRADECIMIENTOS:}

Los autores agradecen a Aguas \& Aguas (empresa de servicios públicos de acueducto y alcantarillado de Pereira) por su apoyo a la investigación.

\section{REFERENCIAS}

[1] M. López et al., "El ciclo urbano del agua. Un nuevo modelo de sistema integral de gestión," Ide@sostenible. Espacio de reflexión y comunicación en desarrollo sostenible. vol. 4, pp. 16, [En línea], Disponible: http:/upcommons.upc.edu/revistes/bitstream/2099/3862/1/ gestion_del_agua_cast.pdf, 2007.

[2] M. Rygaard et al., "Increasing urban water self-sufficiency: New era, new challenges," J. Environ. Manag., vol. 92, pp. 185-194, 2011. DOI: http://dx.doi.org/10.1016/j.jenvman.2010.09.009

[3] C. Qi, y N. Chang, "Integrated carbon footprint and cost evaluation of a drinking water infrastructure system for screening expansion alternatives," J. Clean. Prod., vol. 27, pp. 51-63, 2012. DOI: http://dx.doi.org/10.1016/j.jclepro.2012.01.009 
[4] S. Lim et al., "Urban water infrastructure optimization to reduce environmental impacts and costs," J. Environ. Manag., vol. 91, pp. 630-637, 2010. DOI: http://dx.doi.org/10.1016/j. jenvman.2009.09.026

[5] J. Comas y S. Morera, Life cycle assessment and water management-related issues. Girona, España: Quaderns de medi ambient 4, Documenta Universitaria, Universitat de Girona, 2012, $198 \mathrm{p}$.

[6] S. Lundie et al., "Life cycle assessment for sustainable metropolitan water systems planning," Water Sci. Technol., vol. 38, pp. 3465-3473, 2004. DOI: http://dx.doi.org/10.1021/es034206m

[7] D. De Haas et al., "Life Cycle Assessment of the Gold Coast urban water system. Achieving a balance between opposing environmental impacts is a challenge," Water, vol. 38, n. ${ }^{\circ}$, pp. 57-64, [En línea], Disponible: http://www.urbanwateralliance.org.au/publications/journals/ De_Haas_Gold_Coast.pdf, 2011.

[8] J. Stokes y A. Horvath, "Life Cycle Energy Assessment of Alternative Water Supply Systems," Int. J. Life Cycle Assess., vol. 11, n. ${ }^{\circ}$ 5, pp. 335-343, 2006. DOI: http://dx.doi.org/10.1065/ lca2005.06.214

[9] S. Lassaux et al., "Life Cycle Assessment of Water from the Pumping Station to the Wastewater Treatment Plant," Int. J. Life Cycle Assess., vol. 12, n. ${ }^{\circ}$ 2, pp. 118-126, 2007.

[10] E. Friedrich et al., "Environmental life cycle assessments for water treatment processes - A South African case study of an urban water cycle," Water SA, vol. 35, n. ${ }^{\circ}$ 1, pp. 73-84, 2009. DOI: http://dx.doi.org/10.4314/wsa.v35i1.76710

[11] M. Mohamed et al., "Towards sustainability in urban water: a life cycle analysis of the urban water system of Alexandria City, Egypt," J. Clean. Prod., vol. 18, pp. 1100-1106, 2010.

[12] M.J. Amores et al., "Environmental assessment of urban water cycle on Mediterranean conditions by LCA approach,” J. Clean. Prod., vol. 43, pp. 84-92, 2013. DOI: http://dx.doi. org/10.1016/j.jclepro.2012.12.033

[13] G. Barjoveanu et al., "Evaluation of water services system through LCA. A case study for Iasi City, Romania," Int. J. Life Cycle Assess., vol. 19, pp. 449-462, 2013. DOI: http://dx.doi. org/10.1007/s11367-013-0635-8

[14] D. Lemos et al., "Environmental assessment of an urban water system," J. Clean. Prod., vol. 54, pp. 157-165, 2013. DOI: http://dx.doi.org/10.1016/j.jclepro.2013.04.029

[15] M. Nudelman y R. Pérez, "Conceptos para el manejo de la sostenibilidad del ciclo urbano del agua". Presentado en VI Serea, Seminario Iberoamericano sobre sistemas de abastecimiento urbano de agua, Joao Pessoa, Brasil. Disponible: http://www.lenhs.ct.ufpb.br/html/downloads/ serea/6serea/TRABALHOS/trabalhoC \%20(2).pdf, 2006.

[16] O. Jolliet et al., "Impact 2002+: A new life cycle impact assessment methodology". Int. J. Life Cycle Assess., vol. 8, n. ${ }^{\circ}$ 6, pp. 324-330, 2003. DOI: http://dx.doi.org/10.1007/BF02978505 
[17] International Organization for Standardization (ISO), Environmental Management and Life Cycle Assessment, Principles and Framework, ISO/FDIS 1404, International Organization for Standardization, 2006.

[18] International Organization for Standardization (ISO), "Environmental Management and Life Cycle Assessment, Requirements and Guidelines Managements Environmental," ISO/FDIS 14044, 2006.

[19] J. Suárez y G. Navia, "Aporte a la utilización de agua termal como coagulante en el tratamiento de las aguas residuales municipales," Tesis especialización, Universidad Nacional de Colombia, sede Manizales, 2007.

[20] M. Varón, “Agua para uso doméstico: análisis de ciclo de vida y escenarios de manejo ambiental para la ciudad de Pereira, Colombia,” Tesis de maestría, Universidad Tecnológica de Pereira, 2015.

[21] H. Slagstad y H. Brattebø, "Life cycle assessment of the water and wastewater system in Trondheim, Norway - a case study," Urban Water J., vol. 11, pp. 323-334, 2014. DOI: http:// dx.doi.org/10.1080/1573062X.2013.795232 